\title{
APTITUD COMBINATORIA PARA CALIDAD DE SEMILLA DE MAÍCES NORMALES Y DE ALTA CALIDAD DE PROTEÍNA
}

\author{
COMBINING ABILITY FOR NORMAL AND HIGH QUALITY PROTEIN MAIZE SEEDS
}

\author{
Margarito Manjarrez Salgado $^{1 *}$, Sergio A. Rodríguez Herrera ${ }^{1}$, Noel O. Gómez Montiel ${ }^{2}$, \\ Ernesto Moreno Martínez ${ }^{3}$, Mario E. Vázquez Badillo ${ }^{1}$, Víctor M. Zamora Villa ${ }^{1}$ y Alfonso López Benítez ${ }^{1}$
}

\footnotetext{
${ }^{1}$ Departamento de Fitomejoramiento, Universidad Autónoma Agraria Antonio Narro. 25315, Buenavista, Saltillo, Coahuila. Tel. y fax. 01 (844) $411-0228$. 2 Programa de maíz, Campo Experimental Iguala, Instituto Nacional de Investigaciones Forestales, Pecuarias y Agrícolas. Apartado Postal No. 29. 40000 , Iguala, Guerrero. Tel. y fax. 01 (733) 332-1056. ${ }^{3}$ Facultad de Estudios Superiores de Cuautitlán, Unidad de Investigación de Granos y Semillas, Universidad Autónoma de México. Cuautitlán Izcalli, Estado de México. Tel. 01 (55) 58809316.

*Autor para correspondencia (jeanso57@yahoo.com.mx)
}

\section{RESUMEN}

La asociación entre caracteres agronómicos de campo e indicadores de calidad fisiológica de semillas y sus componentes genéticos, son factores importantes para ampliar la caracterización del germoplasma en un programa de mejoramiento. En esta investigación se analizan los efectos de aptitud combinatoria general (ACG) y específica (ACE) y el tipo de acción génica de los caracteres agronómicos y de calidad fisiológica de la semilla de maíz (Zea mays $L$.) normal y de alta calidad de proteína. El material genético correspondió a 14 líneas endogámicas de maíz, siete de grano normal y siete de alta calidad de proteína. Se analizaron las variables agronómicas: floración masculina y femenina, altura de planta y mazorca, calificación de planta y mazorca, y rendimiento de grano. La calidad fisiológica fue determinada por la germinación estándar inicial y final, longitud de plúmula inicial y final, y peso seco final. Los resultados mostraron variación significativa entre genotipos $(P \leq 0.05)$ en los efectos de ACG y ACE para todas las variables. Se identificaron a las cruzas: B-41 x CML144, CML-144 x CLQ-6203 y LT-154 x CML-177 con los mayores valores de rendimiento de grano con $1.39,1.37$ y $1.19 \mathrm{t} \mathrm{ha}^{-1}$ respectivamente, y a las cruzas T-45 x CLQ-6203, T-37 x CML-141 y LT154 x CML-177 con altos valores de ACE en la germinación estándar final de 23.2, 22.9 y $14.2 \%$, respectivamente. Las líneas T-45, T-44 y LT-155 mostraron el mayor valor de ACG para rendimiento de grano con 0.41, 0.78 y $0.45 \mathrm{t} \mathrm{ha}^{-1}$, y las líneas CML-144 y LT-155 las de mayor ACG para la variable germinación estándar final con 6 y $6 \%$, respectivamente. El tipo de acción génica que predominó fue la aditiva en todas las características agronómicas y fisiológicas.

Palabras clave: Zea mays, grano normal y alta calidad de proteína, aptitud combinatoria general y específica.

\section{SUMMARY}

Association among agronomic field traits and physiological seed quality and their genetic components are important factors for broadening germoplasm characterization in a plant breeding program. This research analyzed the general (GCA) and specific (SCA) combining abilities effects and the genetic action type of agronomic traits and physiological quality of normal and high quality protein seed in maize (Zea mays $\mathbf{L}$.). The genetic material was a group of 14 maize inbred lines, seven of normal grain and seven of high protein quality. The agronomics traits analyzed were: male and female flowering, plant and ear height, plant and ear visual score and grain yield. Physiological quality was determined by initial and final standard germination, initial and final plumule length, and final dry weight. Results showed significative variation among genotypes $(\mathrm{P} \leq$ 0.05) for GCA and SCA effects in all traits. Crosses B-41 x CML-144, CML-144 x CLQ-6203 and LT-154 x CML-177 outstanded by their highest grain yield with $1.39,1.37$ and $1.19 \mathrm{t} \mathrm{ha}^{-1}$ respectively, while crosses T-45 x CLQ-6203, T-37 x CML-141 and LT-154 x CML-177 had the highest SCA values in final standard germination, with 23.2, 22.9 and $14.2 \%$ respectively. Lines T-45, T-44 and LT-155 showed the highest GCA values for grain yield, with $0.41,0.78$ y $0.45 \mathbf{t ~ h a}^{-1}$, while lines CML-144 and LT-155 showed the highest GCA values for final standard germination with $6 \%$ in both cases. The predominating genetic action type was additive for all of the agronomic and physiological traits.

Index words: Zea mays, normal and high quality protein of grain, specific and general combining ability.

\section{INTRODUCCIÓN}

En México existen 31 millones de personas con desnutrición, de los cuales 18 millones padecen desnutrición severa, diez millones en el medio rural y el resto es población urbana de escasos ingresos (Morris y López, 2000). Estos mismos autores mencionan que el maíz (Zea mays L.) es fundamental en la alimentación de los mexicanos, ya que se estima un consumo anual de $209.8 \mathrm{~kg}$ por persona. La proteína en maíz es pobre en lísina y triptofano, aminoácidos esenciales para un buen desarrollo físico y mental del ser humano; de esta manera, se considera de suma importancia el descubrimiento del gen $\operatorname{opaco}_{2}(O$, $)$ el cual se ha incorporado a maíces mejorados, junto con genes modificadores del grano, lo que ha dado lugar a maíces de alta calidad de proteína (Vasal, 1994). El gen $O_{2}$ incrementa la cantidad de estos aminoácidos en las diferentes fracciones del endospermo del maíz, cuyo principal efecto radica en 
reducir la cantidad de zeina y aumentar la proporción de otras proteínas como globulinas y glutelinas (Jiménez, 1966).

Es importante estudiar las causas de los problemas que restringen el uso de maíces de alta calidad proteínica (MACP); entre ellos están la pérdida de viabilidad de las semillas y su deterioro en el almacén por sus propios procesos fisiológicos, los que seguramente están ligados a su constitución genética particular. Este conocimiento permitirá tomar decisiones para hacer un manejo adecuado de los MACP, y a los fitomejoradores seleccionar los genotipos idóneos para obtener híbridos y variedades con semillas tolerantes a la pérdida de viabilidad y vigor durante su manejo postcosecha. El mejoramiento de estos maíces, al aminorar la pérdida postcosecha de la calidad biológica, permitiría su aceptación en los canales comerciales, con el consiguiente beneficio para la agricultura y para el mejoramiento de la dieta del mexicano.

El principal objetivo del mejoramiento genético es generar variedades e híbridos que tengan un alto potencial de rendimiento y características agronómicas deseables basadas en el interés del agricultor. También es necesario producir semillas de alta calidad con respecto a sus componentes genéticos, físicos, fisiológicos y sanitarios. En el aspecto fisiológico de la semilla se ha promovido el desarrollo del concepto vigor como un parámetro del potencial real de la misma, ya que se manifiesta en cuatro etapas del ciclo de vida de las plantas: 1) mayor sobrevivencia en el almacenamiento, 2) mayor emergencia en campo, 3) mejor establecimiento de plántulas, y 4) mayor rendimiento (Woodstock, 1973).

La constitución genética de la semilla interviene como un elemento diferencial en la calidad de la misma, ya que en varias especies se ha demostrado que existe una correlación entre la longevidad y el componente genético de la semilla, incluso entre lotes y aún entre los mismos lotes de semilla (Moreno et al., 1978). Sin embargo, los mecanismos genéticos que determinan el vigor de la semilla han sido poco estudiados.

Dada la relevancia que tiene el conocimiento de estos mecanismos para los investigadores en tecnología de semillas, así como para los fitomejoradores, es importante conocer la variación genética para los diferentes caracteres agronómicos al iniciar cualquier programa de mejoramiento genético, ya que la respuesta a la selección depende de la heredabilidad y de la presión de selección entre materiales (Chávez, 1995). Esta investigación tiene como objetivo determinar la aptitud combinatoria general y específica de progenitores normales y de alta calidad de proteína de maíz, así como identificar el tipo de acción génica más impor- tante en varias características agronómicas y fisiológicas de la semilla.

\section{MATERIALES Y MÉTODOS}

El material genético consistió de14 líneas endogámicas de maíz que fueron utilizadas como progenitoras; siete eran de grano normal, proveniente del Instituto Nacional de Investigaciones Forestales, Agrícolas y Pecuarias (INIFAP): 1) $\mathrm{T}-45$, 2) $\mathrm{T}-44$, 3) $\mathrm{LT}-155$, 4) $\mathrm{B}-41,5) \mathrm{Y}-$ $902314,6) \mathrm{T}-37$ y 7) LT-154, y siete eran de alto contenido proteínico, generadas en el Centro Internacional de Mejoramiento de Maíz y Trigo (CIMMYT): 8) CML-141, 9) CML-142, 10) CML-144, 11) CML-150, 12) CML176, 13) CML-177 y 14) CLQ-6203.

La investigación se desarrolló en dos etapas; la generación y evaluación de las cruzas se hizo en el Campo Experimental del INIFAP en Iguala, Guerrero, ubicado a $17^{\circ} 52^{\prime} 54^{\prime \prime}$ 'LN y $98^{\circ} 45^{\prime} 25^{\prime \prime}$ LO; a una altitud de 780 $\mathrm{m}$, con temperatura media anual de $27.5{ }^{\circ} \mathrm{C}$ y precipitación anual de 977 mm (García, 1973). La determinación de calidad de semilla se llevó a cabo en el Laboratorio de Ensayo de Semillas, del Centro de Capacitación y Desarrollo de Tecnología de Semillas (CCDTS) de la Universidad Autónoma Agraria Antonio Narro (UAAAN), Saltillo, Coah., México y en la Unidad de Investigación de Granos y Semillas (UNIGRAS) de la Facultad de Estudios Superiores de Cuautitlán (FES-C) de la Universidad Nacional Autónoma de México (UNAM).

La obtención de las progenies $\mathrm{F}_{1}$ se realizó durante los ciclos primavera-verano (P-V) 2001/01 y otoño-invierno (O-I) 2001/02, con la finalidad de obtener semilla suficiente. La evaluación del ciclo agrícola P-V 2002/02, hecha bajo un diseño experimental látice triple $10 \times 10$, incluyó las 91 cruzas directas posibles entre las siete líneas de grano normal $(\mathrm{N})$ y las siete de alto contenido proteínico $(\mathrm{C})$, que generaron $21 \mathrm{NN}, 49 \mathrm{NC}$ y $21 \mathrm{CC}$, más nueve testigos regionales; la unidad experimental fue de cuatro surcos de $6 \mathrm{~m}$ de largo y $80 \mathrm{~cm}$ de ancho, para cosechar como parcela útil a los dos surcos centrales de $5.0 \mathrm{~m}$ de largo con 22 plantas cada uno, en una superficie de $8 \mathrm{~m}^{2}$. Se fertilizó con la fórmula $120 \mathrm{~N}-60 \mathrm{P}-00 \mathrm{~K}$; el control de maleza y plagas se hizo de acuerdo con las recomendaciones del INIFAP (Gómez et al., 1995); la cosecha de la parcela útil se hizo a los 125 d después de la siembra, cuando la semilla tenía $18 \%$ de humedad; la semilla se ajustó a $12 \%$ de humedad, y el rendimiento por parcela se transformó a $\mathrm{t}$ $\mathrm{ha}^{-1}$. Además del rendimiento de grano, se obtuvo información de días a floración masculina y femenina (DFM; DFF), altura de planta (AP), altura de mazorca (AM), calificación de la planta (CP) y calificación de mazorca $(\mathrm{CM})$. El análisis de varianza del diseño genético se 
realizó bajo el diseño experimental de bloques completos al azar con tres repeticiones, solamente con las cruzas directas $\mathrm{F}_{1}$.

La prueba de germinación estándar inicial (GSI) se hizo de acuerdo con las normas establecidas por la International Seed Testing Association (ISTA, 1996), mediante el método de papel toalla modificado a cuatro repeticiones de 25 semillas (debido a la poca semilla disponible) tomadas al azar por cada tratamiento (cruza), y el papel se enrolló a manera de "tacos", los cuales fueron colocados en cámara de germinación a $25 \pm 1{ }^{\circ} \mathrm{C}$ por $7 \mathrm{~d}$. Al final se hizo el conteo único de plántulas normales iniciales (PNI), plántulas anormales iniciales (PAI), semillas muertas iniciales (SMI), longitud media de plúmula inicial (LMPI) y peso seco inicial (PSI). Se utilizó un diseño completamente al azar con cuatro repeticiones y sólo se analizaron las cruzas $\mathrm{F}_{1}$.

La prueba de envejecimiento acelerado (EA) se hizo en una cámara de envejecimiento artificial a $35 \pm 1{ }^{\circ} \mathrm{C}$ por 8 d, con 250 semillas colocadas en canastillas de plástico, las cuales se introdujeron en charolas con $2 \mathrm{~L}$ de solución de KCL $85 \%(\mathrm{p} / \mathrm{v})$, sostenidas por un soporte de malla galvanizado en el interior con el fin de que las semillas no estuvieran en contacto con la solución; la caja se cubrió con su respectiva tapa de plástico. Al concluir el periodo de envejecimiento, las semillas se secaron perfectamente y enseguida se sometieron a la prueba de germinación estándar final (GSF), en la que se registraron las cantidades de plántulas normales finales (PNF), plántulas anormales finales (PAF) y de plántulas muertas finales (PMF), así como longitud media de plúmula final (LMPF) y peso seco de plúmula final (PSPF). Se utilizó un diseño completamente al azar con cuatro repeticiones, y solamente se incluyeron las cruzas $\mathrm{F}_{1}$.

Las variables antes citadas se analizaron mediante el modelo IV de Griffing (1956) cuyo modelo lineal es: Yij $=\mu+g i+g j+S i j+y k+$ eijk, donde: Yijk $=$ Valor del fenotipo observado de la cruza; $\mu=$ Media general; gi, gj = Efecto de ACG del progenitor i, j; Sij = Efecto de la ACE de cruza ij; yk = Efecto de la k-ésima repetición; eijk $=$ Efecto del error experimental.

Mediante la esperanza de los cuadrados medios se estimaron la varianza aditiva $\left(\sigma^{2} \mathrm{~A}\right)$, varianza de dominancia $\left(\sigma^{2} \mathrm{D}\right)$, error estándar de ACG (EE $\left.\mathrm{ECG}_{\mathrm{AC}}\right)$ y error estándar de ACE (EEACE), y se calculó la heredabilidad en sentido estricto $\left(\mathrm{h}^{2}\right)$, de la forma siguiente:

$$
h^{2}=\sigma^{2} A / \sigma^{2} P
$$

La varianza fenotípica fue calculada como: $\sigma_{\mathrm{p}}^{2}=\sigma_{\mathrm{A}}^{2}+\sigma_{\mathrm{D}}^{2}+$ CMerror, donde: $\mathrm{P}=$ Número de padres; $\mathrm{r}=$ Número de repeticiones; $\mathrm{CM}_{\mathrm{ACG}}=$ Cuadrado medio de ACG; $\mathrm{CM}_{\mathrm{ACE}}=$ Cuadrado medio de ACE; y CMERror $=$ Cuadrado medio del error.

Las varianzas y errores se calcularon:

$$
\begin{aligned}
& \sigma_{A C G}^{2}=C M_{A C G}-C M_{A C E} / r(P-2) \\
& \sigma_{A}^{2}=2 \sigma^{2}{ }_{A C G} \\
& \sigma_{A C E}^{2} \equiv C M_{A C E}-C M_{e r r o r} / r \\
& \sigma_{D}^{2}=\sigma_{A C E}^{2} \\
& \left.E E_{A C G}=\sqrt{2}-1 / P(P-1)\right][\text { CMerror } / r] \\
& \left.E E_{A C E}=\sqrt{P} P^{2}+P+2 /(P+1)(P+2)\right][\text { CMerror } / r]
\end{aligned}
$$

Todos los análisis se hicieron con el programa de SAS versión 8.0 (SAS Institute, 1999).

\section{RESULTADOS Y DISCUSIÓN}

\section{Variables de campo}

Se detectaron diferencias estadísticas significativas $(\mathrm{P} \leq$ 0.01) entre híbridos, así como en los efectos de aptitud combinatoria general (ACG) y aptitud combinatoria específica (ACE), para todas las variables estudiadas (Cuadro 1). La proporción relativa de los efectos de ACG y ACE determinada por los cuadrados medios, indicó que el tipo de acción génica más importante correspondió a los efectos de ACG, en concordancia con lo descrito por Baker (1978).

\section{Variables fisiológicas}

También se detectaron diferencias significativas $(\mathrm{P} \leq$ 0.01) para casi todas las variables fisiológicas en estudio (Cuadro 2). Con base en la proporción de los cuadrados medios, los efectos aditivos (ACG) fueron más importantes que los efectos no aditivos (ACE), en casi todas las variables de calidad fisiológica, excepto en germinación estándar inicial (GSI) cuyo cuadrado medio fue de 236 y el de ACG que tuvo un valor de 234, y ambos tipos de acción génica fueron significativos lo que indica que ambos tipos de efecto se pueden explotar. 
Cuadro 1. Cuadrados medios para el análisis de varianza genético para características agronómicas evaluadas en Iguala, Gro. PV $2002 / 02$.

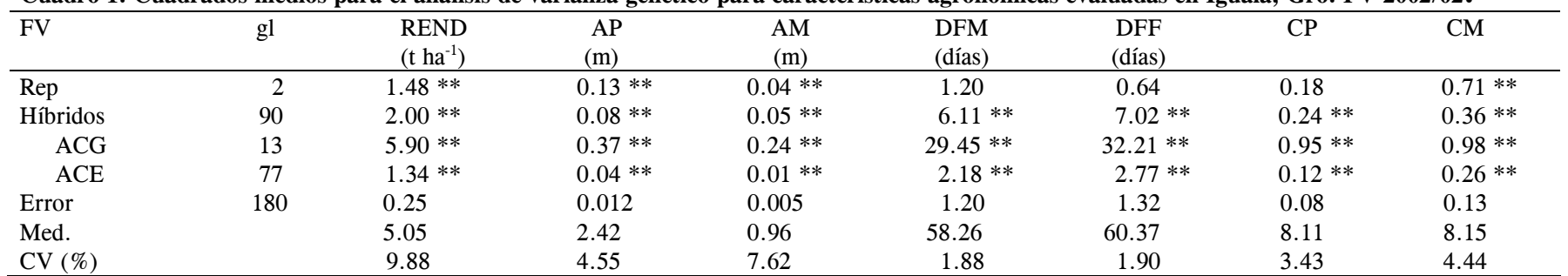

$*, * *=$ Significativo a 5 y $1 \%$ de nivel de significancia, respectivamente.

REND = Rendimiento; AP = Altura de planta; AM = Altura de mazorca; DFM = Días a floración masculina; DFF = Días a floración femenina; CP = Calificación de planta; $\mathrm{CM}=$ Calificación de mazorca, escala visual 1 a 10; $\mathrm{CV}=$ Coeficiente de variación; gl = Grados de libertad; FV = Fuente de variación.

Cuadro 2. Cuadrados medios del análisis de varianza para características fisiológicas de semillas en 2003.

\begin{tabular}{lrccccc}
\hline FV & gl & $\begin{array}{c}\text { GSI } \\
(\%)\end{array}$ & $\begin{array}{c}\text { LMPI } \\
(\mathrm{cm})\end{array}$ & $\begin{array}{c}\text { GSF } \\
(\%)\end{array}$ & $\begin{array}{c}\text { LMPF } \\
(\mathrm{cm})\end{array}$ & $\begin{array}{l}\text { PSPF } \\
(\mathrm{mg})\end{array}$ \\
\hline Híbridos & 90 & $236.6^{* *}$ & $5.3^{* *}$ & $453.5 * *$ & $9.6^{* *}$ & $0.06 * *$ \\
ACG & 13 & $234.7^{* *}$ & $13.0 * *$ & $740.8^{* *}$ & $31.6 * *$ & $0.13 * *$ \\
$\quad$ ACE & 77 & $237.0 * *$ & $4.0 * *$ & $405.0 *$ & $5.9 * *$ & $0.05 * *$ \\
Error & 273 & 19.0 & 0.7 & 47.0 & 1.1 & 0.01 \\
Media & & 94.6 & 8.0 & 80.0 & 7.3 & 0.36 \\
CV $(\%)$ & & 4.6 & 10.4 & 8.6 & 14.9 & 14.5 \\
\hline
\end{tabular}

$*, * *=$ Significativo a 5 y $1 \%$ de nivel de significancia, respectivamente. GSI = Germinación estándar inicial; LMPI = Longitud media de plúmula inicial; GSF $=$ Germinación estándar final; $\mathrm{LMPF}=$ Longitud media de plúmula final; $\mathrm{PSPF}=$ Peso seco plúmula final; $\mathrm{CV}=$ Coeficiente de variación; $\mathrm{gl}=$ Grados de libertad; $\mathrm{FV}=$ Fuente de variación.

\section{Aptitud combinatoria general}

Variables agronómicas. En los valores estimados de ACG para las variables agronómicas (Cuadro 3) se identificó que para rendimiento de grano, cinco de los progenitores de grano normal tuvieron valores positivos y solamente dos presentaron valores negativos; en los materiales de alta calidad de proteína sucedió lo contrario, ya que seis líneas tuvieron valores negativos y solamente una dio valor positivo. La línea T-44 de grano normal con los valores más altos de ACG en rendimiento de grano, presentó características agronómicas deseables en altura de planta y mazorca, días a floración masculina y femenina y calificación de planta y mazorca; en cambio, CML-144 de alta calidad de proteína y única línea con valores positivos para rendimiento de grano, mostró valores indeseables en todas las características agronómicas.

Variables fisiológicas. En las variables germinación estándar inicial (GSI) y final (GSF), consideradas como las de mayor importancia para determinar la calidad de la semilla, las líneas CML-144 y CML-150 de alta calidad de proteína, y B-41, LT-155 y Y-902314 de grano normal, tuvieron los valores mayores de ACG en germinación estándar inicial (Cuadro 4). Pero al ser sometidas al estrés de la prueba de envejecimiento acelerado (prueba de vigor), algunos materiales se deterioraron, como fue el caso de la línea CML-150 de alta calidad de proteína; lo contrario se observó en la línea CML-144, que soportó el estrés y mantuvo un valor positivo para la prueba de vigor. El mismo fenómeno se presentó en los materiales de grano normal, ya que la línea LT-155 soportó el estrés mientras que la línea B-41 se deterioró durante la prueba de vigor. Para la variable longitud de plúmula final sucedió algo similar, pues las líneas CML-144, LT-155 y B-41 soportaron mejor la prueba de vigor. Se notó un equilibrio de valores positivos (4) y negativos (3), tanto en las líneas de calidad de proteína como en los maíces normales, en cuanto a germinación estándar inicial (GSI) y final (GSF). Es decir, los datos obtenidos no apoyan el postulado que los materiales de alta calidad de proteína pierden más rápido su vigor y poder de germinación que los maíces normales.

Cuadro 3. Efectos de aptitud combinatoria general (ACG) para las variables agronómicas en Iguala, Gro. PV 2002/02.

\begin{tabular}{llccccccc}
\hline & Línea & $\begin{array}{c}\text { REND } \\
\left.(\mathrm{t} \mathrm{ha})^{-1}\right)\end{array}$ & $\begin{array}{l}\text { AP } \\
(\mathrm{m})\end{array}$ & \multicolumn{1}{c}{$\begin{array}{l}\text { AM } \\
(\mathrm{m})\end{array}$} & $\begin{array}{l}\text { DFM } \\
(\text { días })\end{array}$ & $\begin{array}{r}\text { DFF } \\
(\text { días })\end{array}$ & CP & CM \\
\hline 1 & T-45 & 0.42 & -0.04 & -0.02 & -1.12 & -1.04 & 0.22 & 0.31 \\
2 & T-44 & 0.78 & -0.15 & -0.10 & -0.84 & -1.09 & 0.28 & 0.17 \\
3 & LT-155 & 0.45 & -0.01 & -0.02 & -1.20 & -1.31 & 0.14 & -0.11 \\
4 & B-41 & -0.03 & -0.02 & -0.02 & 0.49 & 0.16 & -0.04 & -0.12 \\
5 & Y-902314 & -0.20 & 0.04 & 0.04 & 0.80 & 0.85 & 0.04 & 0.23 \\
6 & T-37 & 0.23 & 0.08 & 0.04 & 0.58 & 0.96 & -0.01 & 0.06 \\
7 & LT-154 & 0.32 & -0.08 & -0.08 & 1.19 & 1.16 & 0.13 & -0.01 \\
8 & CML-141 & -0.32 & 0.08 & 0.11 & 0.16 & 0.05 & -0.23 & -0.19 \\
9 & CML-142 & -0.24 & 0.13 & 0.15 & 0.88 & 0.83 & -0.33 & -0.16 \\
10 & CML-144 & 0.17 & 0.11 & 0.09 & 0.41 & 0.46 & -0.07 & 0.08 \\
11 & CML-150 & -0.70 & -0.19 & -0.15 & 0.58 & 0.52 & -0.04 & -0.26 \\
12 & CML-176 & -0.23 & 0.13 & 0.01 & 0.30 & 0.71 & -0.01 & 0.06 \\
13 & CML-177 & -0.31 & -0.04 & -0.01 & -1.73 & -1.56 & -0.07 & 0.02 \\
14 & CLQ-6203 & -0.34 & -0.04 & -0.06 & -0.48 & -0.70 & -0.01 & -0.09
\end{tabular}

REND $=$ Rendimiento $;$ AP $=$ Altura de planta: $\mathrm{AM}=$ Altura de mazorca; DFM $=$ Días a floración masculina; DFF $=$ Días a floración femenina; $\mathrm{CP}=$ Calificación de planta; $\mathrm{CM}=$ Calificación de mazorca;

Nota: Del 1 al 7 son maíces de grano normal, y del 8 al 14 son de alta calidad de proteína. 
Cuadro 4. Efectos de aptitud combinatoria general para las variables fisiológicas. PV 2003.

\begin{tabular}{llccccc}
\hline & Línea & $\begin{array}{c}\text { GSI } \\
(\%)\end{array}$ & $\begin{array}{c}\text { LMPI } \\
(\mathrm{cm})\end{array}$ & $\begin{array}{c}\text { GSF } \\
(\%)\end{array}$ & $\begin{array}{c}\text { LMPF } \\
(\%)\end{array}$ & $\begin{array}{c}\text { PSPF } \\
(\%)\end{array}$ \\
\hline 1 & T-45 & -5.69 & -0.89 & -3.00 & -1.59 & -0.10 \\
2 & T-44 & -1.02 & 0.57 & 2.67 & -0.13 & -0.04 \\
3 & LT-155 & $\mathbf{1 . 5 6}$ & 0.58 & $\mathbf{6 . 0 0}$ & 0.82 & -0.02 \\
4 & B-41 & $\mathbf{2 . 2 3}$ & 1.06 & -1.17 & 1.96 & 0.12 \\
5 & Y-902314 & $\mathbf{1 . 4 8}$ & 0.18 & $\mathbf{0 . 6 7}$ & 0.56 & 0.04 \\
6 & T-37 & 0.14 & 0.37 & -7.00 & -0.30 & -0.04 \\
7 & LT-154 & -0.52 & -0.36 & 1.67 & -0.23 & 0.03 \\
8 & CML-141 & 0.14 & -0.05 & 0.00 & 0.29 & -0.05 \\
9 & CML-142 & -0.77 & -0.51 & 4.08 & -0.01 & 0.01 \\
10 & CML-144 & $\mathbf{2 . 3 9}$ & 0.08 & $\mathbf{6 . 0 0}$ & 0.20 & 0.04 \\
11 & CML-150 & $\mathbf{2 . 2 3}$ & -0.26 & -4.83 & -0.59 & 0.02 \\
12 & CML-176 & 0.31 & -0.36 & 0.08 & -0.22 & 0.03 \\
13 & CML-177 & -2.94 & 0.01 & -0.92 & -0.04 & -0.01 \\
14 & CLQ-6203 & 0.48 & -0.42 & -4.25 & -0.72 & -0.03 \\
\hline
\end{tabular}

GSI = Germinación estándar inicial; LMPI $=$ Longitud media de plúmula inicial; GSF = Germinación estándar final; LMPI = Longitud media de plúmula final; PSPF $=$ Peso seco plúmula final.

Nota: Del 1 al 7 son maíces de grano normal y del 8 al 14 son de alta calidad de proteína; las cifras en negritas indica líneas sobresalientes para GSI y GSF

\section{Aptitud combinatoria específica}

Variables agronómicas. De las 91 cruzas directas, 44 presentaron valores positivos y 47 valores negativos para la variable rendimiento de grano. En el Cuadro 5 se muestran las 15 cruzas con los mayores valores de ACE y tres con los más bajos; tales datos indica que existe un efecto positivo al cruzar maíces normales con los de calidad de proteína; sin embargo, la cruza 10 x 14 que tiene dos líneas de alta calidad de proteína, dio un valor estimado positivo de 1.37 para rendimiento de grano y valores estimados negativos para altura de planta y mazorca, días a floración masculina y femenina, que son los deseables para obtener híbridos de porte bajo, precoces y con buena calificación de planta y mazorca. También las cruzas B-41 x CML144, LT-154 x CML-177, LT-155 x CML-176, LT-154 x CML-141 sobresalieron con valores estimados de ACE para rendimiento de grano de $1.39,1.19,0.99$ y $0.98 \mathrm{tha}^{-1}$ respectivamente, en las cuales participa una línea de maíz normal por una línea de alta calidad de proteína, que son el tipo de materiales deseados porque combinan la longevidad del maíz normal y alta calidad de proteína del maíz mutante.

Variables fisiológicas. Las mejores 15 cruzas simples en germinación estándar final, considerada como la variable de mayor importancia para determinar el vigor en cada híbrido, se muestran en el Cuadro 6. De las 91 cruzas, 54 tuvieron valores positivos y 37 valores negativos; algunos materiales tuvieron buena germinación, como las cruzas T-45 x CLQ-6203, T-37 x CML-141, LT-154 x CML177, T-45 x CML-176, T-44 x Y-902314, T-44 x CML141 y T-45 x Y-902314, con valores estimados de ACE de $23.1,22.9,14.1,13.8,10.5,8.2$ y $5.2 \%$, respectivamente, las cuales al ser sometidas a estrés mediante la prueba de envejecimiento acelerado, también mostraron tolerancia. Los híbridos tolerantes correspondieron a las cruzas entre una línea de grano normal por una línea de alta calidad de proteína, excepto T-44 x Y-902314 y T-45 x Y902314. En general, la combinación de líneas de grano normal por las de calidad de proteína resistieron más el estrés, por lo que sería conveniente obtener este tipo de maíces con mayor longevidad en almacén.

Cuadro 5. Efectos genéticos de aptitud combinatoria específica de las cruzas sobresalientes de 91 evaluadas, en las variables agronómicas Iguala, Gro. PV 2002/02.

\begin{tabular}{cccccccc}
\hline Cruza & $\begin{array}{l}\left.\text { REND }_{(\mathrm{t} \mathrm{ha}}^{-1}\right) \\
\text { AP }\end{array}$ & $\begin{array}{c}\text { AM } \\
(\mathrm{m})\end{array}$ & $\begin{array}{c}\text { DFM } \\
(\text { días })\end{array}$ & $\begin{array}{c}\text { DFF } \\
(\text { días })\end{array}$ & CP & CM \\
\hline $4 \times 10$ & $\mathbf{1 . 3 9}$ & 0.22 & 0.11 & -0.16 & -0.32 & 0.16 & 0.23 \\
$10 \times 14$ & $\mathbf{1 . 3 7}$ & -0.07 & -0.03 & -0.19 & -0.46 & 0.30 & 0.70 \\
$7 \times 13$ & $\mathbf{1 . 1 9}$ & 0.27 & 0.16 & -0.39 & -0.63 & 0.33 & 0.34 \\
$3 \times 12$ & $\mathbf{0 . 9 9}$ & -0.01 & 0.02 & 0.31 & -0.10 & 0.26 & 0.40 \\
$7 \times 8$ & $\mathbf{0 . 9 8}$ & 0.08 & 0.05 & 0.06 & 0.43 & -0.01 & -0.12 \\
$5 \times 14$ & 0.94 & 0.01 & 0.02 & -1.58 & -1.52 & 0.02 & -0.12 \\
$6 \times 11$ & 0.93 & -0.10 & 0.02 & -0.08 & -0.18 & 0.27 & 0.38 \\
$1 \times 14$ & 0.93 & 0.09 & 0.05 & 0.00 & 0.04 & 0.34 & 0.30 \\
$2 \times 9$ & 0.88 & 0.11 & 0.04 & -0.30 & -0.43 & 0.27 & 0.17 \\
$5 \times 9$ & 0.78 & 0.04 & 0.04 & -0.28 & -0.71 & 0.01 & 0.29 \\
$3 \times 13$ & 0.71 & -0.01 & 0.01 & 0.00 & -0.16 & -0.02 & 0.10 \\
$5 \times 11$ & 0.68 & 0.02 & 0.03 & -0.64 & -0.74 & 0.05 & 0.38 \\
$2 \times 4$ & 0.67 & -0.02 & 0.07 & -0.25 & -0.43 & -0.02 & 0.13 \\
$2 \times 6$ & 0.65 & 0.01 & 0.00 & -0.33 & -0.57 & -0.05 & -0.05 \\
$11 \times 14$ & 0.61 & 0.09 & 0.06 & -0.03 & -0.52 & -0.23 & 0.20 \\
$\ldots$ & & & & & & & \\
$7 \times 11$ & -1.46 & -0.16 & -0.01 & 1.64 & 1.62 & -0.37 & -0.55 \\
$7 \times 14$ & -1.51 & 0.03 & -0.03 & 1.36 & 1.18 & -0.23 & -0.55 \\
$3 \times 5$ & -1.77 & -0.15 & -0.11 & 1.14 & 0.76 & -0.13 & -0.27 \\
\hline
\end{tabular}

REND $=$ Rendimiento; AP $=$ Altura de planta AM $=$ Altura de mazor ca; $\mathrm{DFM}=$ Días a floración masculina; $\mathrm{DFF}=$ Días a floración femenina; $\mathrm{CP}=$ Calificación de planta; $\mathrm{CM}=$ Calificación de mazorca.

Nota: Las cifras en negritas indica cruzas sobresalientes para REND.

Los maíces de calidad de proteína rindieron $1.13 \mathrm{t} \mathrm{ha}^{-1}$ en promedio, significativamente inferiores a los maíces de grano normal; sin embargo, al cruzarlos con un material normal su rendimiento aumentó en $0.7 \mathrm{t} \mathrm{ha}^{-1}(\mathrm{P} \leq 0.05)$ por lo que es posible explotar esta heterosis y las ventajas que pudieran tener en su aprovechamiento para elote y su mayor rendimiento en masa (Cuadro 7). En las características fisiológicas los maíces normales cruzados con un material de alta calidad de proteína superaron $(\mathrm{P} \leq 0.05)$ a los materiales de calidad de proteína y a los materiales de 
grano normal, en la variable germinación estándar final que representa el vigor de los materiales, así como en longitud y peso seco de la plúmula (Cuadro 8).

Cuadro 6. Efectos genéticos de aptitud combinatoria específica para las cruzas sobresalientes de 91 evaluadas, para las variables fisiológicas. PV 2003.

\begin{tabular}{|c|c|c|c|c|c|}
\hline Cruza & $\begin{array}{l}\text { GSI } \\
(\%)\end{array}$ & $\begin{array}{l}\text { LMPI } \\
(\mathrm{cm})\end{array}$ & $\begin{array}{l}\text { GSF } \\
(\%)\end{array}$ & $\begin{array}{l}\text { LMPF } \\
(\mathrm{cm})\end{array}$ & $\begin{array}{r}\text { PSPF } \\
\text { (mg) }\end{array}$ \\
\hline $1 \times 10$ & 8.74 & 2.10 & -1.08 & -0.01 & -0.07 \\
\hline $2 \times 13$ & 8.40 & 0.52 & -4.83 & -0.43 & -0.09 \\
\hline $1 \times 6$ & 6.99 & 1.26 & -24.08 & -1.61 & -0.11 \\
\hline $1 \times 8$ & 6.99 & 0.87 & -5.08 & 0.08 & 0.03 \\
\hline $7 \times 13$ & 6.90 & 0.84 & 14.17 & 0.96 & 0.18 \\
\hline $2 \times 8$ & 6.32 & 1.19 & 8.26 & 0.73 & 0.05 \\
\hline $1 \times 12$ & 5.82 & 0.10 & 13.84 & 1.58 & 0.26 \\
\hline $1 \times 5$ & 5.65 & 0.17 & 5.26 & 0.00 & 0.01 \\
\hline $4 \times 13$ & 5.15 & 1.02 & -2.99 & -1.07 & 0.05 \\
\hline $11 \times 13$ & 5.15 & 0.15 & -4.33 & 0.74 & -0.04 \\
\hline $2 \times 5$ & 4.99 & 0.94 & 10.59 & -2.08 & -0.15 \\
\hline $5 \times 13$ & 4.90 & -0.44 & -1.83 & -2.44 & -0.10 \\
\hline $1 \times 14$ & 4.65 & 1.15 & 23.17 & 2.05 & 0.16 \\
\hline $6 \times 8$ & 4.15 & 0.48 & 22.92 & -0.59 & -0.03 \\
\hline $6 \times 9$ & 4.07 & 0.86 & 0.84 & -0.97 & 0.05 \\
\hline $8 \times 10$ & -11.10 & -2.23 & -8.08 & -0.05 & -0.10 \\
\hline $2 \times 6$ & -29.68 & -2.30 & -16.74 & 1.29 & -0.03 \\
\hline $1 \times 13$ & -48.93 & -2.94 & 0.84 & 0.85 & 0.06 \\
\hline
\end{tabular}

GSI = Germinación estándar inicial; LMPI $=$ Longitud media de plúmula inicial; GSF = Germinación estándar final; LMPI = Longitud media de plúmula final; PSPF $=$ Peso seco plúmula final.

Nota: Las cifras en negritas indica cruzas sobresalientes para GSF.

Cuadro 7. Promedio de características agronómicas de híbridos de grano normal $x$ normal $(\mathbf{N} \times \mathbf{N})$, normal $x$ calidad de proteína $(\mathbf{N} \times \mathbf{C})$ y calidad de proteína $x$ calidad de proteína $(C \times$ C), en Iguala,Gro. PV 2002.

\begin{tabular}{lccccccc}
\hline $\begin{array}{l}\text { Núm. } \\
\text { Cruzas }\end{array}$ & $\begin{array}{l}\text { REND } \\
\left(\mathrm{t} \mathrm{ha}^{-1}\right)\end{array}$ & $\begin{array}{l}\text { AP } \\
(\mathrm{m})\end{array}$ & $\begin{array}{l}\text { AM } \\
(\mathrm{m})\end{array}$ & $\begin{array}{l}\text { DFM } \\
(\text { días })\end{array}$ & $\begin{array}{l}\text { DFF } \\
(\text { días })\end{array}$ & CP & CM \\
\hline $21 \mathrm{NN}$ & $5.54 \mathrm{a}$ & $2.34 \mathrm{~b}$ & $0.90 \mathrm{~b}$ & $58.2 \mathrm{a}$ & $60.3 \mathrm{a}$ & $8.3 \mathrm{a}$ & $8.3 \mathrm{a}$ \\
$49 \mathrm{NC}$ & $5.12 \mathrm{~b}$ & $2.45 \mathrm{a}$ & $0.97 \mathrm{a}$ & $58.3 \mathrm{a}$ & $60.4 \mathrm{a}$ & $8.1 \mathrm{ab}$ & $8.2 \mathrm{ab}$ \\
$21 \mathrm{NC}$ & $4.41 \mathrm{c}$ & $2.44 \mathrm{a}$ & $0.98 \mathrm{a}$ & $58.3 \mathrm{a}$ & $60.4 \mathrm{a}$ & $7.9 \mathrm{~b}$ & $8.0 \mathrm{~b}$ \\
\hline
\end{tabular}

Letras iguales indican ausencia de diferencias significativas a 0.01 y 0.05 de probabilidad de error (t- Student)

REND $=$ Rendimiento; AP = Altura de planta; $\mathrm{AM}=$ Altura de mazorca; DFM $=$ Días a floración masculina; DFF $=$ Días a floración femenina; $\mathrm{CP}=$ Calificación de planta; $\mathrm{CM}=$ Calificación de mazorca.

Cuadro 8. Promedio de características fisiológicas de híbridos de grano normal $x$ normal $(N \times N)$, normal $x$ calidad de proteína $(N \times C)$ y calidad de proteína $\mathrm{x}$ calidad de proteína $(\mathrm{C} \times \mathrm{C}$ ) en Iguala, Gro. PV 2003.

\begin{tabular}{lccccl}
\hline $\begin{array}{l}\text { No. } \\
\text { Cruzas }\end{array}$ & $\begin{array}{l}\text { GSI } \\
(\%)\end{array}$ & $\begin{array}{l}\text { LMPI } \\
(\mathrm{cm})\end{array}$ & $\begin{array}{l}\text { GSF } \\
(\%)\end{array}$ & $\begin{array}{l}\text { LMPF } \\
(\mathrm{cm})\end{array}$ & $\begin{array}{l}\text { PSF } \\
(\mathrm{mg})\end{array}$ \\
\hline $21 \mathrm{NN}$ & $93.19 \mathrm{a}$ & $8.17 \mathrm{a}$ & $76.95 \mathrm{~b}$ & $7.52 \mathrm{a}$ & $0.32 \mathrm{~b}$ \\
$49 \mathrm{NQ}$ & $94.88 \mathrm{a}$ & $8.20 \mathrm{a}$ & $82.71 \mathrm{a}$ & $7.38 \mathrm{a}$ & $0.39 \mathrm{a}$ \\
$21 \mathrm{QQ}$ & $94.71 \mathrm{a}$ & $7.15 \mathrm{~b}$ & $77.05 \mathrm{~b}$ & $6.95 \mathrm{~b}$ & $0.34 \mathrm{~b}$
\end{tabular}

Letras iguales indican ausencia de diferencias significativas al $0.01 \mathrm{y}$ 0.05. $(\mathrm{t}$ - Student). GSI $=$ Germinación estándar inicial; LMPI $=$ Longitud media de plúmula inicial; GSF = germinación estándar final; LMPI $=$ Longitud media de plúmula final; PSPF $=$ Peso seco plúmula final.

\section{Heredabilidad}

La heredabilidad $\left(\mathrm{h}^{2}\right)$ para las características agronómicas presentó valores intermedios, con excepción de la variable altura de planta que tuvo una heredabilidad alta y de calificación de mazorca que tuvo una heredabilidad baja. En todas las variables fisiológicas se obtuvo una heredabilidad baja, con excepción de la longitud de plúmula final que mostró una heredabilidad intermedia (Cuadro 9). Aunque no existe una escala definida para clasificar la magnitud de la heredabilidad, Robles (1986) considera que un valor bajo es de 0 a 0.3 , uno medio de 0.3 a 0.7 y uno alto de 0.7 a 1.0; en cambio, Chávez (1993) señala que no está bien definido lo que se entiende por alta heredabilidad. En general son aceptables los siguientes valores: alta heredabilidad, mayor de 0.5; heredabilidad media, de 0.2 a 0.5 ; y baja heredabilidad, menos de 0.2 , en función del carácter en estudio.

Cuadro 9. Determinación de la heredabilidad a través de las componentes de varianza de variable agronómicas y fisiológicas evaluadas en 2002 y 2003. Iguala, Gro.

\begin{tabular}{lccccc}
\hline Variables & Va & Vd & ESVa & ESVd & h2 \\
\hline REND & 0.25 & 0.36 & 0.12 & 0.07 & 0.29 \\
AP & 0.02 & 0.01 & 0.01 & 0.00 & 0.47 \\
AM & 0.01 & 0.00 & 0.00 & 0.00 & 0.60 \\
DFM & 1.52 & 0.30 & 0.60 & 0.12 & 0.50 \\
DFF & 1.64 & 0.48 & 0.65 & 0.15 & 0.48 \\
CP & 0.05 & 0.01 & 0.02 & 0.01 & 0.33 \\
CM & 0.04 & 0.04 & 0.02 & 0.01 & 0.19 \\
GSI & -0.10 & 54.48 & 3.90 & 9.44 & 0.00 \\
LMPI & 0.38 & 0.85 & 0.20 & 0.16 & 0.20 \\
GSF & 13.99 & 89.56 & 11.59 & 16.14 & 0.09 \\
LMPF & 1.07 & 1.22 & 0.48 & 0.24 & 0.32 \\
PSF & 0.00 & 0.01 & 0.00 & 0.00 & 0.21 \\
\hline Va Vd & & &
\end{tabular}

$\mathrm{Va}, \mathrm{Vd}=$ Valores estimados de varianza aditiva y dominancia; $\mathrm{h} 2=$ Valores estimados de heredabilidad; ESVa , ESVd = Valores estimados de errores estándar de varianza aditiva y dominancia.

\section{CONCLUSIONES}

Las cruzas B-41 x CML-144, CML-144 x CLQ-6203, LT-154 x CML-177 tuvieron los mayores valores estimados de ACE para rendimiento de grano con 1.39, $1.37 \mathrm{y}$ $1.19 \mathrm{t} \mathrm{ha}^{-1}$, respectivamente y las cruzas T-45 x CLQ6203, T-37 x CML-141, LT-154 x CML-177 tuvieron altos valores de ACE para la germinación estándar final, de 23.2, 22.9 y $14.2 \%$, respectivamente. Las líneas T-45, T44 y LT-155 mostraron los mayores valores de ACG para rendimiento, y las líneas CML-144 y LT-155 fueron las de mayor ACG para germinación estándar final. 
Los cruzamientos normal $\mathrm{x}$ normal fueron mejores estadísticamente que las cruzas normal x calidad, y éstas fueron mejores estadísticamente que los maíces calidad x calidad para rendimiento de grano y para la mayoría de las características agronómicas y fisiológicas.

La heredabilidad para rendimiento de grano fue intermedia con 0.29 , pero para la germinación estándar final la heredabilidad fue baja, con un valor de 0.09. El tipo de acción génica que predominó en las características agronómicas y fisiológicas estudiadas fue la aditiva.

\section{BIBLIOGRAFÍA}

Baker R J (1978) Issues in diallel analysis. Crop Sci. 18:533-536.

Chávez A J L (1993) Mejoramiento de Plantas 1. Ed. Trillas. México, D.F. $136 \mathrm{p}$.

Chávez A J L (1995) Mejoramiento de Plantas 2. Métodos Específicos de Plantas Alógamas. Ed. Trillas. México, D.F. 143 p.

García E (1973) Modificaciones al sistema de clasificación climatológica de Köppen para adaptarlo a las condiciones de la República Mexicana. Instituto de Geografía. Universidad Nacional Autónoma de México. México, D. F. 217 p.
Gómez M N, P Murillo N, S Navarro G, R Cruzaley S (1995) Guía para la Asistencia Técnica, Campo Agrícola Experimental del CEIGUA. Instituto Nacional de Investigaciones Forestales y Agropecuarias. Secretaría de Agricultura, Ganadería y Desarrollo Rural. Iguala, Gro. México. pp:15-23.

Griffing B (1956) Concept of general and specific combining ability in relation to diallel crossing systems. Aust. J. Biol. Sci. 9:463:493.

International Seed Testing Association, ISTA (1996) International Rules for Seed Testing. Rules Seed Sci. Technol. 24 (Suppl.): 241-335.

Jiménez J R (1966) Protein fraction studies of high lisine corn. In: Proc. High Lysine Corn Conference. Corn Industries Research Foundation. Washington, D. C. pp:74-79.

Moreno M E, R Morones R, L Gutiérrez R (1978) Diferencias entre líneas, cruzas simples y dobles de maíz en su susceptibilidad al daño por condiciones adversas de almacenamiento. Turrialba 28:233-237.

Morris M L, M A López P (2000) Impactos del mejoramiento de maíz en América Latina 1996-1997. CIMMYT. México D.F. 45 p.

Robles S R (1986) Genética Elemental y Fitomejoramiento Práctico. Ed. Limusa. México. D. F. 477 p.

SAS Institute (1999) SAS Language and Procedure Usage. Version 8. SAS Institute Inc., Cary, N. C.

Vasal S K (1994) High quality protein corn. In: Specialty Corns. A.R. Hallauer (ed). CRC Press. Boca Ratón, FL. USA. pp:79-121.

Woodstock L W (1973) Physiological and biochemical test for seed vigor. Seed Sci. Technol. 1:127-157. 